\title{
Unravelling Transmission in Epidemiological Models and its Role in the Disease-Diversity Relationship
}

\author{
Marjolein E.M. Toorians ${ }^{1, *}$, Ailene MacPherson ${ }^{2}$, and T. Jonathan Davies ${ }^{1,3,4}$ \\ ${ }^{1}$ Department of Botany, Biodiversity Research Centre, University of British Columbia, 2212 Main \\ Mall, Vancouver, BC V6T 1Z4, Canada \\ ${ }^{2}$ Department of Mathematics, Simon Fraser University, Burnaby, Canada \\ ${ }^{3}$ African Centre for DNA Barcoding, University of Johannesburg, Johannesburg 2092, South \\ Africa \\ ${ }^{4}$ Department Forest \& Conservation Sciences, University of British Columbia, 2212 Main Mall, \\ Vancouver, BC V6T 1Z4, Canada \\ ${ }^{*}$ Corresponding author
}

\begin{abstract}
There are a multitude of pathways a pathogen can invade and spread through a host population. The assumptions of the transmission model used to capture disease propagation determines the outbreak potential, the pathogen's net reproductive success $\left(R_{0}\right)$. This review offers an insight into the assumptions and motivation behind common transmission mechanisms, and introduces a general framework with which we unify all, where contact rate, the most important parameter in disease dynamics, determines the type of transmission model. This general transmission model framework helps bridge the gap between mathematical disease modelling and the much debated disease-diversity relationship, by expanding common disease models to multiple hosts systems, and in considering the role of host diversity in disease transmission. By describing the mechanisms of transmission as a stepwise process, we provide a guide for modelling pathogens in multi-host systems and elaborate how these transmission mechanisms can affect host communities, but also how host communities affect the pathogen's success. We further expand on these models and introduce an approach for including host species' evolutionary history into transmission dynamics to hereafter aid the debate on the effect of biodiversity and community composition on disease outbreak potential.
\end{abstract}

Keywords: Epidemiological Models, Transmission, Biodiversity, Dilution Effect

* Marjolein.toorians@botany.ubc.ca 


\section{Introduction}

Worldwide, many natural populations are declining rapidly due to anthropogenic influences (IPBES, 2020), resulting in a shift in the abundance of humans and livestock relative to wildlife species (Bar-On et al., 2018). Simultaneously, we are seeing an increase in disease outbreaks originating from wildlife (Daszak et al., 2000; Jones et al., 2008; Smith et al., 2014). Demographic changes have led to increasing contact between humans and livestock with wildlife, providing new opportunities for disease spillover (Graham et al., 2008). Most emerging pathogens in humans are directly transmitted viruses or bacteria that have crossed the species barrier, perhaps multiple times, likely facilitated by changes to the environment made by humans (Dobson and Foufopoulos, 2001; Smith et al., 2014). To understand how recent biodiversity losses and the increasing abundance of humans and domesticated species have reshaped the disease landscape, we need to consider the role of multi-species communities in the maintenance and onward transmission of diseases (Keesing et al., 2010) and the establishment of pathogens in novel hosts.

Transmission describes the process by which an infected individual transmits a pathogen to an uninfected individual, and is a critical step in disease outbreak and spillover (Park et al., 2018). When interspecific transmission is sufficiently high, a pathogen may invade the novel host population (Daszak et al., 2000; Fenton and Pedersen, 2005; Wolfe et al., 2007). An example of disease spillover is shown in Figure 1. A key strategy for preventing spillover is to reduce transmission from the reservoir (a population of a single species that can maintain the disease) to a susceptible, novel target population (Haydon et al., 2002). Understanding the process of transmission within and between species is thus an essential step for mitigating the risk of future disease spillover and emergence events.

Transmission is a complex, stepwise process (see Box 1 and Figure 1); however, underlying assumptions are opaque, often hidden within a single composite parameter. In the susceptible-infected-recovered (SIR) compartmental model (Kermack and McKendrick, 1927; Anderson and May, 1982), the focal model for this paper, the transmission process is frequently simplified as being density-dependent, density-independent or some intermediate between these two extremes. However, transmission is determined not only by the inherent behaviour of the pathogen, but also by that of the host or vector, as most pathogens rely on their host for reproduction (Rohani et al., 2003; Han et al., 2015). Properties of the host population that affect disease transmission include contact rates, population density, and individual birth and death rates, whereas other disease parameters, such as recovery and transmission rates are considered pathogen dependent parameters (Han et al., 2020). In addition, transmission is unlikely a homogeneous process, but integrates across many heterogeneities at different stages of transmission, including host contact rates and susceptibility (McCallum et al., 2017; Smith et al., 2009). Nonetheless, in many epidemiological models, transmission is represented as a simple, singular constant.

The choice of transmission model affects the projected disease dynamics, and thus the potential management decisions that follow. It is critical, therefore, to choose the model that best represents the pathogen life-history and host population behaviour. Here, we synthesize assumptions underlying common transmission models, and how they affect the dynamics of the simple compartmental (SIR) model of infectious disease spread. In particular, we will explore the effect of host contact rate and structure on disease transmission and show it is the most important determinant of these variations. While limited in epidemiological complexity, focusing on this subset of models allows us to examine details of the transmission process relevant for inter-specific disease transmission, spillover and the much debated disease-diversity relationship (Halliday and Rohr, 2019). 


\section{Box 1: The Biology of Transmission}

Transmission is the transfer of infectious particles, or propagules, from an infected (donor) host, to a naive, receiving host. Transmission potential is then dependent on a threshold propagule load within the donor host (Wilber et al., 2016; Blaser et al., 2014). As pathogen particles often have to travel through space between leaving the donor host and establishing in the recipient host, the environment and the time a particle is free-roaming can play a role in transmission success (Tien and Earn, 2010). McCallum et al. (2017) deconstructed transmission into five discrete stages:

- Stage 1: Dynamics of propagules within donor host.

- Stage 2: Production of pathogen-infective stages in donor host.

- Stage 3: Pathogen survival and growth in the environment (including the environment of an intermediate host).

- Stage 4: Dose acquired by recipient host at exposure.

- Stage 5: Pathogen load in the recipient host.

Between these stages of transmission, heterogeneities can arise and threshold-like behaviours may emerge. Conventional, linear frameworks of transmission may overlook such heterogeneity, for example, super-spreaders resulting from high infection loads (McCallum et al., 2017). For instance, nematode crowding within hosts causes an increased immune response, and saturates the relationship between transmission Stages 1 and 2, such that at high pathogen levels the production of the dispersal pathogen (nematode) life-stage plateaus (McCallum et al., 2017). Age of infection, host immunosuppressive capabilities and pathogen competition (in case of co-infection) can also have an effect on the production of pathogen dispersal stages (Stage 2). An example is Malaria, where physiological traits affect the susceptibility of individuals: People with sickle cell anaemia are more resistant to the disease (Elguero et al., 2015). In diseases such as cholera, high pathogen loads cause diarrhoea and vomiting and therefore increased shedding of infectious particles.

For Stage 3, the environment determines the subsequent received load, but the donor/recipients host behaviour is of importance too, determining the exposure to the shed pathogen. Some dispersal stages can linger outside hosts longer than others. For example, the decay rate of Mycobacteria, causing tuberculosis, is dependent on the temperature of the medium and likely also to light-exposure, therefore differing among seasons (Fine et al., 2011). However, some pathogens (e.g. HIV) are never exposed to the environment, and are directly transmitted, these pathogens skip Stage 3 .

The load the recipient host acquires depends on its exposure (Stage 4), which is impacted by multiple factors including: the relative density of the donor and recipient hosts, the survival rate of the free-living pathogen stage, and, for trophically transmitted pathogens, the Holling type II function response.

The final load that establishes in the recipient host (Stage 5) depends on the quality of the received particle, the host immune response (which may depend on its genotype), and the presence of other pathogens (co-infection). These stages can differ between each pathogen, complicating their simplification into standard models. Figure 1 shows these transmission stages in the spillover of the bacterium bovine Tuberculosis (bTB) from the reservoir host (African buffalo, Syncerus caffer), to a novel host (elephant, Loxodonta africana) (Miller et al., 2021). This example emphasises the complex dynamics underlying transmission.

A key challenge in modeling transmission, and the focus of this manuscript, is capturing the inherent nature of host density and contact rates. Contacts cannot indefinitely increase with population density, as is assumed in some models (Smith et al., 2009). Contact structure and animal behaviour can have a large impact on disease dynamics, as shown in bTB models for buffalo by Cross et al. (2004, 2007), where allowing for social groupings broke down standard SIR disease dynamics. Another illustration is provided by the European badger, also a reservoir for bTB, 
where culling intended to reduce number of infected individuals instead resulted in an increased contact rate (as aggregation increased in lower-density groups), and thus increased disease prevalence (Vicente et al., 2007). The epidemiological contact process, and the emergent relationship between host density and contact rate, can be modeled both explicitly, in the form of density-dependent or density-independent compartmental models, and implicitly, for example, as a network or individual-based model. Here, we focus on the representation of transmission in the SIR compartmental model. We provide a step-by-step guide to decomposing transmission, and introduce a novel contact rate-function that allows us to unify existing models. We examine how the biology of transmission and the mathematical assumptions in our models inform our understanding of the disease-diversity relationship. Finally, we present a novel approach for including host species' identities (evolutionary history) into our framework, adding to our understanding of host community structure and biodiversity on disease outbreak potential.

\section{Box 2: The transmission-virulence trade-off}

Pathogen load is often used as a proxy for virulence, the pathogen-induced host mortality. A higher pathogen load also translates to a higher rate of transmission (Box 1 - stage 2), as there are more dispersal propagules (McCallum, 2016; Wilber et al., 2016). For successful transmission, a sufficient number of propagules need to be produced within the donor host, survive the intermediate stages, and establish in the recipient host. When virulence is high, transmission may be reduced due to the negative effect of high pathogen load on host behaviour, for example, high fevers in influenza immobilizes patients, reducing pathogen spread (Dieckmann et al., 2005), and a pathogen that kills its host quickly reduces its window of opportunity for infection. This is known as the transmission - virulence trade-off (Cressler et al., 2016). However, pathogen load is not always a good predictor of transmission success. For example, HIV has highest transmission potential at intermediate viral loads (McKay et al., 2020), and the trade-off between transmission and virulence has increasingly been questioned.

\section{Decomposing the transmission function, $F(S, I)$}

In the classic SIR model, the transmission rate, $\beta$, describes the rate of new infections per unit of time. It is often modeled as a single constant, but can represent a composite of many different underlying processes. These dynamics are described by the transmission function, $F(S, I)$, encompassing $S$ and $I$ individuals and the rate of transmission, $\beta$, which has been described in numerous formulations (see Table 1). The transmission term can also be described as the product of the force of infection (often referred to as $\lambda$ ) and the density of susceptible individuals (de Jong et al., 1995). The transmission function is represented in the SIR model in the derivative for $S$ and $I$ as:

$$
\begin{aligned}
& \frac{d S}{d t}=-F(S, I) \\
& \frac{d I}{d t}=F(S, I)-\Gamma I
\end{aligned}
$$

Where $t$ is time and $\Gamma$ the removal rate (recovery and mortality rates, combined). $S$ and $I$ are the number of susceptible and infected individuals, respectively. Throughout this paper the host population size is defined as $N=S+I+R$, unless specified otherwise. Assuming infection is a probability, the rate of infection can be described as (Begon et al., 2002):

$$
F(S, I)=\beta S \frac{I}{N}
$$

where $\beta$ is the transmission rate which determines the spread of infection from infected $(I)$ to susceptible $(S)$ hosts. There are several equally valid formulations of $F$, but we will focus on the one presented in Equation (2) as it is most common in ecology. The force of infection is defined as $\lambda=\beta \frac{I}{N}$. For infection events to happen on the same scale as recovery events in System (1), $\lambda$ must be of order $O(1)$ such that $F(S, I)$ is always of $O(N)$.

Here we decompose commonly used transmission functions, $F(S, I)$, of compartmental SIR models (Table 1). We begin with the different relationships between susceptible and infected individuals for homogeneously and heterogeneously mixing populations (not including explicit contact networks) (Table 1) and finish with explaining how the 
contact rate $(\kappa)$ determines the density or frequency-dependent nature of transmission, together with the probability of successful transmission $(c)$. We provide a schematic illustrating our framework in Figure 2, starting with standard homogeneity (left column) and finishing with some examples of heterogeneity (right column). Table 2 summarizes the parameters that are used here and in the equations throughout this review.

In the model of System 1, the criterion for a disease outbreak is proportional to the transmission rate. Namely, the conditions under which a disease can spread is determined by the basic reproductive rate, $R_{0}$, which describes the number of secondary infections arising from a single infected host in a fully susceptible host population. This quantity is also known as the intrinsic lifetime reproductive success or fitness of the pathogen. It tells us the pathogen's lifetime reproductive success early on in an infection, as the quantity growth rate tells us the growth rate in the absence of competition in a model of logistic growth. As shown in Box 3, in an SIR model with density-dependent transmission $R_{0}$ can be simply defined as:

$$
R_{0}=\frac{\beta}{\Gamma}
$$

Emergence of disease, or pathogen persistence in a community, will occur when $R_{0}>1$. If $R_{0}<1$, the pathogen will be lost from the host community, whereas an $R_{0}$ equal to 1 represents an endemic disease. In addition to determining disease emergence, Equation (3) describes the intrinsic rate of increase of the pathogen.

\subsection{Homogeneity}

We start our decomposition of the transmission function assuming homogeneous populations (Figure 2, left column). There are various different options for modelling the interaction between susceptible and infected individuals, and a pathogen's transmission rate, $\beta$ (McCallum et al., 2001; Begon et al., 2002; Hoch et al., 2008). However, all formulations of $\beta$ depend on the contact rate $(\kappa)$, as given by the average number of contacts per host per unit time, and the probability of infection given contact $c$. Here, we first derive the expression for $\beta$ in terms of $\kappa$ and $c$ and then introduce a novel general contact rate function $\kappa(N)$ from which we then derive a range of transmission rates commonly used in the literature, see Table 1 for a summary.

\section{Decomposing transmission rate, $\beta$}

In a homogeneous population, the rate at which new infections occur is given by:

$$
\beta=-\kappa \cdot \ln (1-c),
$$

see Keeling and Rohani (2011) Box 2.1 for a formal derivation. This formulation of the transmission rate assumes that the number of contacts per unit time $\kappa$ is constant over time and hence density-independent. This assumption is often the case for sexually transmitted diseases and is violated, for example, in cases where public-health interventions directly alter host contact. When the probability of infection given contact, $c$, is small, this equation can be simplified into the more intuitive form of the product of the contact rate, $\kappa$, and the proportion of those contacts resulting in infection, $c$, (McCallum et al., 2001, 2017). Then, $\beta$ can be defined simply as:

$$
\beta=\kappa \cdot c
$$

see Appendix for a formal derivation. Using this linear relationship between $\kappa$ and $c$, we can then determine the $R_{0}$ of a disease as:

$$
R_{0}=\kappa \cdot c \cdot \tau
$$

where $\tau$ is the duration of the infection. This formulation can be used to approximate $R_{0}$ from empirical data (Anderson et al., 1992). The dynamics of the transmission function, $F(S, I)$, are dependent on the type of contacts between hosts, as described in the rate $\kappa$, such that, combining Equation (2) and Equation (5):

$$
F(S, I)=\beta S \frac{I}{N}=\kappa c S \frac{I}{N}
$$




\section{General contact rate}

The dynamics of $\kappa$ determine the density-dependent (DD) or frequency-dependent (FD) nature of $F(S, I)$. Here we present a generalized version of this contact rate that can be adapted to reveal the different natures of the transmission functions, $F(S, I)$, described in Table 1 :

$$
\kappa(N)=\frac{\xi}{\omega} N^{q}
$$

The factor $\xi$ is a constant (contacts per unit time), which determines the metric of transmission that will be held constant across population of different size. $\omega$ portrays the biotic or abiotic asymptotic effects of the transmission. If one wants to create heterogeneity in classes, $F(N)$ (and therefore also $\kappa(N)$ ) can be extended to be $F(S, I, R$ ) (and $\kappa(S, I, R)$ ), such that classes can have different biotic effects via the parameter $\omega_{S}, \omega_{I}$ and $\omega_{R}$. Parameter $q$ determines whether the transmission rate is $\mathrm{DD}(q=1)$, FD $(q=0)$ or some intermediate between these (Smith et al., 2009) (See Figure 3).

\subsubsection{Frequency-Dependence}

The simplest form of transmission is Frequency-Dependence, FD, where the proportion of the population each host interacts with per unit time, $\xi$, is held constant regardless of host population size. Setting $\omega=1$ and $q=0$ in Equation (8), such that the contact rate is constant $(\kappa=\xi)$. As a result, the transmission rate is independent of host population density:

$$
\beta_{F}=\kappa \cdot c=\xi \cdot c
$$

Substituting this into Equation (2), we return the familiar FD transmission function (Row 1 of Table 1):

$$
F(S, I)=\xi c S \frac{I}{N}=\beta_{F} \frac{S I}{N} \quad \text { where } \beta_{F}=\xi c
$$

Here, infections rise with the probability $\left(\frac{I}{N}\right)$, the density of infected hosts. Note that $\xi$, and hence $\kappa$ is $O(N)$. A key assumption in FD is the constant number of contacts per time unit. Common examples of FD transmission include sexually transmitted diseases for which transmission relies on the limited number of contacts or 'transmission events' between sexual partners. Vectored diseases, such as Lyme's disease, have also been approximated assuming FD, with transmission dependent on contact with an intermediate host. In this example, the vector determines the speed of transmission, which is limited by, for example, the number of meals the vector has per time unit. The FD force of infection dynamic is shown in Figure 4A.

If populations remain near their carrying capacity, a particularly useful approximation of FD transmission can be obtained by using the carrying capacity $(K)$, instead of population size: $F(S, I)=\frac{\beta_{F} S I}{K}$ (Row 5 , Table 1 ). This reduces mathematical complexity by removing the dependence of the dynamics on a ratio of state variables, $S$ and I. This dynamic, here referred to as Carrying Capacity Dependent transmission, is shown in Figure 4D.

\subsubsection{Density-dependence}

The DD-transmission function is often referred to as (pseudo-) mass action, where population size can change but density remains constant (the fraction of contacts is constant) (Kermack and McKendrick, 1927). This model assumes that the quantity $\xi$ is the proportion of the population each individual comes into contact with per unit time and unit area. Hence the resulting number of total interactions per host scales linearly with population density. This model is therefore most appropriate when encounters between individuals are assumed to be brief (Begon et al., 2002; Smith et al., 2009). Setting $\omega=A$ and $q=1$ in Equation (8) $\kappa$ becomes host density-dependent, which is simply expressed as:

$$
\kappa(N)=\frac{\xi N}{A}
$$


Here, the number of contact events grows linearly with the density of hosts (Anderson and May, 1982; Antonovics, 2017). We can therefore say that in this model, $\omega=A$ determines the abiotic limitations on disease spread through area. Combining Equation (2) and Equation (11), the term $N$ is canceled:

$$
\begin{aligned}
F(S, I) & =\kappa c S \frac{I}{N} \\
& =\frac{\xi N}{A} c S \frac{I}{N} \\
& =\xi \cdot c \frac{S I}{A} \\
& =\beta_{A} \frac{S I}{A} \quad \text { where } \beta_{A}=c \xi
\end{aligned}
$$

This brings us to the second and third equations in Table 1 . Note that here $\beta_{D}$ is an order of magnitude higher than $\beta_{F}$. It is of importance to notice that here, $\beta$ has to be of $O\left(\frac{1}{N}\right)$ to satisfy the $F(S, I)$ to be of $O(N)$. This is represented in the units for $\beta_{D}$ (see Table 1). Additionally, $\beta_{A}$ includes area in its units. This can be useful for modelling wildlife populations, as area is important in moderating population behaviour (e.g. aggregation and dispersal) in response to density changes (Civitello et al., 2018).

Often times area is simply considered as a constant $(A=1)$, which further simplifies the the transmission function to:

$$
F(S, I)=\beta_{D} S I
$$

This returns our third equation in Table 1. It is assumed that with constant area, an increase in number of individuals causes an increase in density, hence density-dependence (Kermack and McKendrick, 1927). This transmission model (Figure 4B) can fit well to directly transmitted diseases, such as the common cold or influenza, but is most commonly used for modeling wildlife diseases,including rabies virus in reservoir dogs or tuberculosis in possums (White et al., 2017; Rhodes et al., 1998; Barlow, 1991).

\section{Comparison between DD and FD transmission}

The per-capita transmission in FD $\left(\hat{\beta}=\frac{1}{S I} \frac{d I}{d t}\right)$ declines with increasing population size (Ferrari et al., 2011), as illustrated in the measles outbreak in England (Bjørnstad et al., 2002), where increasing city size decreased mean $\beta$, as predicted by FD dynamics. In contrast, $\hat{\beta}$ for DD is independent of population size. Therefore, in FD, $R_{0}$ remains constant across varying $N$, but increases with $N$ in DD (Ferrari et al., 2011). One consequence of this difference is that DD models predict a critical population density as an invasion threshold (See Box 3), which is not the case for FD.

\subsubsection{Intermediate DD-FD}

Host contact behaviour is often somewhere on the DD-FD continuum (Anderson et al., 1992; Smith et al., 2009; Ferrari et al., 2011). For example, it is unlikely that contact rates either increase indefinitely with N, as is assumed under DD, or is completely density independent, as assumed in FD (McCallum et al., 2001). The general contact rate function, defined above, can be adapted to capture intermediate DD-FD transmission, with the moderating parameter $q(0<q<1)$ and setting $\omega=0$ in Equation (8) such that:

$$
\kappa(N)=\xi N^{q}
$$

where the dimensions for $\xi$ are individuals ${ }^{(-q)}$ and $A$ was set to 1. Combining Equation (14) with Equation (2), brings us: 


$$
\begin{aligned}
F(S, I) & =\kappa c S \frac{I}{N} \\
& =\xi N^{q} c S \frac{I}{N} \\
& =c \xi S I \frac{N^{q}}{N} \\
& =c \xi S I N^{(q-1)} \\
& =\beta_{q} S I N^{(q-1)} \quad \text { where } \beta_{q}=c \cdot \xi
\end{aligned}
$$

When $q=0, N$ drops out and contact rate is again independent of $N$, thus $q=0$ is FD, and $q=1$ is DD. We can interpret $q$ as the relative importance of a single added host within a population to the average contact rate. Now, the order of $F(S, I)$ is $O\left(\frac{1}{N^{q}}\right)$, therefore neither fully DD nor FD, which is represented in the units of $\beta_{q}$ of ind ${ }^{-q} t^{-1}$ (See Table 1). Thus, it that at values of $0<q<0.5, \beta_{q}$ has FD units $\left(t^{-1}\right)$, and at values $0.5<q<1$, DD units $\left(\right.$ ind $\left.d^{-1} t^{-1}\right)$. Likewise, $\xi$ changes in units due to this switch from FD to DD with increasing $q$. This formulation was presented by Smith et al. (2009) to describe cowpox in voles, where $q$ could also vary seasonally (more FD in late summer and more DD in late winter). Note that by using our unifying contact function (Eqn 8), we have flipped the definition of $q$ from the original paper (Smith et al., 2009), however the function remains the same. These intermediate FD-DD dynamics are displayed in Figure 4C.

\subsubsection{Asymptotic transmission}

Some diseases are more likely to spread asymptotically, such that at low host densities contacts are directly proportional to host density (DD), but a maximum rate of contact is obtained at high host densities (e.g. due to spatial or social distribution) (Diekmann and Kretzschmar, 1991; McCallum et al., 2001). Including an additional term for the saturation of contacts at high population sizes, we can model these dynamics by allowing transmission to vary between DD and FD depending on the total population size (Antonovics, 2017). When $\omega=1+\frac{X}{N}$ and $q=1$ in Equation (8), we can derive an asymptotic contact rate function:

$$
\kappa(N)=\frac{\xi}{1+\frac{X}{N}}
$$

where $X$ is the critical population size. The behaviour of the contact function is determined in relation to population size, $N$. When $N \approx 0, \kappa \approx 0$ and transmission approximates DD dynamics, and when $N \approx \infty, \kappa(N) \approx \xi$ and transmission is FD, so shifting from DD to FD with increasing $N$. The transmission function is then:

$$
\begin{aligned}
F(S, I) & =\kappa c S \frac{I}{N} \\
& =\frac{\xi}{1+X N} c S \frac{I}{N} \\
& =c \xi S I \frac{1}{X+N} \frac{1}{N} \\
& =\beta_{F} \frac{S I}{N+X} \quad \text { where } \beta_{F}=\xi c
\end{aligned}
$$

Note, here $\beta$ is identical of the case of frequency dependence, $\beta_{F}$. We can thus define $X=N^{*}$ (with $\left.1<X<\infty\right)$, as the half saturation constant in Michealis-Menten kinetics, which is the critical level after which contacts start to saturate. The lower $X$, the higher the affinity of individual contacts and the quicker saturation of contacts is reached. The $F(S, I)$ is again of $O(N)$. Figure $4 \mathrm{E}$ shows the asymptotic transmission dynamics. 


\section{$2.2 \quad$ Heterogeneity}

The models described above assume individuals are homogeneous in their susceptibility and that epidemiologically relevant contacts between hosts occur at random, known as the assumption of random mixing. However, this is not always the case, for example, a host's behaviour can change the probability of encountering an infected individual and the geographic distribution and movement of hosts plays a significant role in modifying host contact rates (Pope et al., 2007; Vicente et al., 2007). This heterogeneity can affect the overall disease spread (Ferrari et al., 2011), and we thus decompose $F(S, I)$ separately for heterogeneous mixing (Figure 2, right column).

\section{Behavioural heterogeneity}

There are various approaches that allow us to include heterogeneity in behaviour of $S$ and $I$, including network or individual-based models (Ferrari et al., 2011). Here, we review some simplified and commonly adopted formulations for compartmental SIR models.

\subsubsection{Power-law transmission}

This non-linear density-dependent transmission function simulates heterogeneous interactions between $S$ and $I$ individuals with accelerating and decelerating disease spread by raising $S$ and $I$ to the powers $m$ and $n$, respectively (Hochberg, 1991):

$$
F(S, I)=\beta_{P L} S^{m} I^{n}
$$

Parameters $m$ and $n$ determine the speed of infection, and represent how the densities $S$ and $I$ affect the per capita transmission efficiency of the pathogen, such as the susceptibility (by $m$ ) or the infectivity (by $n$ ) of an individual (Hochberg, 1991; Novozhilov, 2008). When both parameters are unity, the model describes simple DD (with constant area). There are many possible response types, as theoretically $-\infty<m, n<\infty$. Some biologically relevant ones are considered further, below.

Assuming $n=1$, when $m>1, F(S, I)$ accelerates, and new infections arise exponentially with linearly increasing number of susceptible individuals. When $0<m, n<1$ and rate of new infections decelerates (Gubbins et al., 2000). Figure $4 \mathrm{~F}$ shows the power law transmission dynamics with varying $n$. We could find no real world examples of exponents $0<m<1$, but it would represent a case when increasing $S$ decreases the rate of transmission (Novozhilov, 2008). Greer et al. (2008) found that Ambystoma tigrinum virus in its host was best described with a Power-law function with $m=1$ and $n=0.255$, suggesting that infection scales positively but non-linearly with $I$. When $m<$ $0, S$ is inversely related to $F(S, I)$, and when $n<0, I$ is inversely related to $F(S, I)$. An example of the latter is seen in Bacillus thuringiensis infections of Plodia interpunctella moths, here, transmission requires cannibalism of infected individuals, and thus transmission increases with $S$, and decrease with $I$ (Knell et al., 1996). When $n=$ 0 , the infection risk is a constant, and independent of the density of infected individuals as is the case where there is constant re-inocculation of the disease from another host, for example, rabies from a bat reservoir host (Greer et al., 2008; Mollentze et al., 2020). By sampling from a distributions of $m$ and $n$, the power-law function can also approximate heterogeneity in transmission rate among $S$ and $I$ individuals (White et al., 2017).

\section{Spatial heterogeneity}

The assumption in SIR models of spatial homogeneous populations is obviously an oversimplification of real population dynamics, especially for species exhibiting territoriality, sociality and other complex group or individual behaviours (Cross et al., 2004; Viana et al., 2014; White et al., 2017). Contact networks provide a useful approach to model more realistic population behaviour (Craft, 2015), but can rapidly become analytically intractable. Here we illustrate how spatial heterogeneity in contacts can be included into our compartmental SIR models, making a few simplifying assumptions, and holding transmission rate, $\beta$, constant. 


\subsubsection{Refuge effect}

The refuge model, as applied to TB in possums, attempts to include spatial aggregation of hosts, with the transmission function incorporating host clustering (Barlow, 1991). In this model, the population consists of patches with and without disease, $p$ is the proportion of the total area that is occupied by diseased individuals, and represents how aggregated the disease is in space. The more the disease is aggregated (the smaller $p$ ), the lower the probability that an infectious individual will encounter a susceptible individual, and thus:

$$
F(S, I)=\beta_{D} I\left(N-\frac{I}{p}\right)
$$

Assuming $N=S+I$, so in this case a simple SI model excluding the $\mathrm{R}$ compartment, the number of susceptible individuals is $S=N p-I$. If $p=1$, the diseased patch equals the total area, there is no aggregation of the disease, and the transmission function simplifies to $F(S, I)=\beta_{D} I(N-I)=\beta_{D} S I$, Equation (13), DD dynamics. With greater aggregation, transmission $F(S, I)$ decreases, as individuals are now less homogeneously distributed. For $I=p N$, which is the maximum density of individuals in the diseased patch, $F(S, I)=0$.

Possums in New Zealand provide an example of aggregated spatial prevalence for TB, due to variation in carrying capacity across habitat patches (Barlow, 1991; May and Anderson, 1984), returning a maximum contact rate per patch, $\beta_{D} K$. Figure 5A shows this transmission dynamic for three different values of $p$.

\subsubsection{Negative binomial}

The negative binomial model builds upon the Refuge effect (Barlow, 1991) and was also fit to model TB in possums, but with varying carrying capacities (different resource availability) per patch, and therefore allowed for increased contact rates by concentrating hosts (Civitello et al., 2018). Here transmission is modelled as:

$$
F(S, I)=\theta S \cdot \ln \left(1+\frac{\beta_{D} I}{\theta}\right)
$$

and includes a heterogeneous mixing term, $\theta\left(\right.$ ind $^{-1} \mathrm{t}^{-1}$ ), with values $0<\theta<\infty$ (Barlow, 2000). Small $\theta$ corresponds to highly aggregated infections. This model assumes that the probability of infection is higher when a susceptible individual has an infected neighbor, and so infections are clustered in space. As $\theta \rightarrow 0, F(S, I)$ decreases as the mean number of infected individuals encountered per susceptible individual is reduced. As $\theta \rightarrow \infty$, aggregation is reduced, and $F(S, I)$ again simplifies to DD transmission. This negative binomial dynamic is shown in Figure 5B.

\subsection{Conclusion transmission functions}

The contact rate between hosts determines the shape of transmission dynamics. There are multiple approaches for describing the spread of a pathogen through a host population, the most common distinction is between DD and FD dynamics, but there are numerous variations, reflecting differences in the biology of the pathogen and the behaviour of the host. For example, wildlife diseases are almost always modeled assuming DD dynamics, regardless of the pathogen, as host behaviour, including local heterogeneities, is more likely to approximate mass-action dynamics at larger scales (White et al., 2017). In contrast, human interactions commonly depart from assumptions of DD, as we model local dynamics (people frequently form small contact networks), and might thus be better modelled using FD dynamics or, more realistically, with asymptotic transmission. However, many empirical studies have shown evidence of both FD and DD dynamics in the same system, with density-dependent dynamics more often observed at low population densities, and frequency-dependent dynamics more common at higher densities (Antonovics, 2017; Roberts and Heesterbeek, 2018). Unsurprisingly, more complex dynamics are found in many disease systems. Within a host population, not all individuals are the same, they may vary in susceptibility and infectivity, captured by the concept of superspreaders, which dramatically influences outbreak potential and disease dynamics (Lloyd-Smith et al., 2005). 
Similar concepts can be extended to super-movers, super-recipients, super-shedders, and super-susceptibles (Streicker et al., 2013; Craft, 2015; White et al., 2017). Additionally, hosts likely do not have a homogeneous contact structure, either through networks or population density. For example, in feline retrovirus, infrequent encounters at low host densities make contact rates close to constant (FD), at intermediate densities contact rate becomes proportional to density (DD), while at high densities there is a further increase in contact rate due to overlapping territories (Fromont et al., 1998). These and other classic case studies, as in the example of how culling of badgers increased intraspecific contacts and tuberculosis prevalence, illustrate how contact structure can be important for modeling wildlife diseases (Vicente et al., 2007; Pope et al., 2007; McCallum, 2016; White et al., 2017). However, contacts in complex networks, such as buffalo, tend to homogenize over time (Cross et al., 2004). Therefore over longer timescales, heterogeneity can average out, providing an opportunity to simplify otherwise complex models.

Box 3: Derivation of the pathogen's net reproductive success, $R_{0}$

To calculate Eqn (3), we want to know when the pathogen can grow in the population, using Eqns 1:

$$
\begin{array}{r}
\frac{d I}{d t}>0 \\
F(S, I)>\Gamma I
\end{array}
$$

Using the the example of density dependent transmission, of Eqn (13), we get:

$$
\frac{\beta S I}{N}>\Gamma I
$$

Then, dividing by $I$ and rearranging we get:

$$
\frac{S}{N}>\frac{\Gamma}{\beta}
$$

So, when $S \sim N$, the disease will spread when $\frac{\beta}{\Gamma}$ is greater than unity, which is the definition of $R_{0}$ :

$$
R_{0}=\frac{\beta}{\Gamma}
$$

\section{Transmission in multi-host systems}

Pathogens that infect only a single host are rare, and it is common for pathogens to infect a number of different host species (Woolhouse et al., 2001), which can significantly alter disease dynamics. Thus, host community structure can both affect and be affected by pathogen dynamics. In multi-host systems, transmission is determined by multiple shedding hosts, each of which can contribute differently to disease prevalence, depending on their inter- and intraspecific ecological interactions (Haydon et al., 2002; Streicker et al., 2013; Fenton et al., 2015). This complicates models and how we estimate transmission, which transmission may be asymmetric among hosts, with some hosts acting as pathogen reservoirs, and pathogens may express different life-history syndromes in different hosts (Haydon et al., 2002; Gandon, 2004). In this section, we describe how multi-host, single-pathogen systems may be influenced by host diversity, the role of transmission, and how transmission can be defined in such systems.

\subsection{Host community dynamics}

The dynamics of multi-host diseases can vary greatly with the species composition of the host community. One important relationship in wildlife disease dynamics is that between disease prevalence and host community diversity. The disease-diversity relationship is a well-known and highly-disputed concept in disease ecology. 


\subsubsection{Host community effects on pathogen prevalence}

The dilution effect suggests higher diversity of hosts reduces the probability of a pathogen infecting a new host, either directly, by reducing encounters, and therefore transmission, between hosts or indirectly, by changing total host abundance (Keesing et al., 2006, 2010) (Table 3). A modelled example of encounter reduction (also known as frequency-dependent dilution) is provided in FD systems by Rudolf and Antonovics (2005), here a host is rescued from pathogen mediated-extinction (apparent mutualism) by a second host that is infected by the same disease. In this model, the secondary host is assumed less competent, and therefore functions as a buffer, reducing further disease spread by replacing contacts (and therefore transmission events) with the original host, effectively reducing frequency of contacts between competent hosts. Lyme disease is a frequently cited example of the dilution effect where diversity of vertebrates hosts decreases the risk of spillover to humans. The bacterial pathogen (Borrelia burgdorferi) is vectored by the black legged tick (Ixodes scapularis), and uses the white footed mouse (Peromyscus leucopus) as a primary host; however, prevalence of B. burgdorferi decreases when a secondary, less competent host, the eastern Chipmunk (Tamias striatus), increases in density (Keesing et al., 2006). Evidence for a dilution effect was also reported for bovine TB in sub-Saharan Africa, which was reduced at higher mammal density (Huang et al., 2013, 2014).

In contrast to the dilution effect, the amplification effect suggests that higher host diversity increases disease prevalence either directly, by elevating contact rates by increasing total host density (Rudolf and Antonovics, 2005) or by the addition of a highly competent, super-spreader host (a phenomenon parallel to the selection effect in biodiversity science (Loreau and Hector, 2001)), or indirectly by changing host densities through competition (Holt and Bonsall, 2017) (see Table 3). For example, higher amphibian diversity is thought to have increased Chytrid disease (caused by Batrachochytrium dendrobatidis) prevalence in some species of frogs, as highly competent (amplifying) hosts are more abundant in species-rich habitats (Ostfeld and Keesing, 2012). The amplification effect may also arise in vectored diseases (often modelled as FD due to density-independent contacts) if increased diversity offers more competent host species so that this multi-host pathogen can more easily persist in the system (Ostfeld and Keesing, 2000). In FD, amplification depends on host identity and, by extension, its competence. Under strict assumptions of DD, amplification will always occur, as contacts increase with host density, and contacts are always additive and never substitutive (Dobson, 2004; Rudolf and Antonovics, 2005).

The direct effect of diversity in DD transmission will always lead to disease amplification, assuming host populations do not compete and the addition of a host is additive to the community. Increasing the number of hosts simply increases encounter rates between $S$ and $I$ individuals, and will therefore always amplify the disease, regardless of the competence of the hosts. Theoretically, assuming strict DD transmission, dilution through direct effects is impossible. In contrast, the direct effect of diversity in FD transmission, assuming a constant number of contacts per time unit and varying competence, are mixed. The addition of a less competent host will always have a diluting effect, whereas the addition of a highly competent host may have an amplifying effect. In Table 3 we summarise how transmission type can cause dilution or amplification. Predicting the indirect effects of diversity is more challenging. Changes in prevalence will reflect changes in the relative abundance of hosts and their relative disease competence, and thus how communities are altered by the introduction of novel host species (Ostfeld and Keesing, 2000). Community evenness is a particularly important dimension in multi-host systems, as the relative dominance of the most competent host will largely determine disease dynamics (Ostfeld and Keesing, 2000; Sintayehu et al., 2017). For example, dilution can occur indirectly in Lyme's disease when the alternative host reduces the density of the main reservoir through competition (Ogden and Tsao, 2009).

In all cases, it is assumed that the pathogen is a generalist and that hosts differ in competence, except for direct amplification in DD systems, which assumes contacts are additive and there is no limit to contacts per time unit. However, these latter assumptions may be unrealistic for many empirical systems (Ostfeld et al., 2008) and, as we discuss above, host contacts likely saturate over higher density Antonovics (2017). Thus, at a certain density, disease dynamics may switch from DD to FD dynamics, and so we might predict dilution effects to be generally more common at higher densities. 


\subsubsection{Pathogen effects on host community}

While we expect a pathogen's persistence to reflect host abundance, host abundance might also be influenced by the pathogen. For example, pathogen sharing among hosts can result in apparent competition (Holt and Pickering, 1985). A reservoir host can indirectly suppress the density of a spillover host by acting as a source of infection, and if the reservoir host has a better adapted immune system to the pathogen, it can reduce the density of the spillover host via pathogen induced mortality. One example of apparent competition is spillover of the vector-transmitted Barley Yellow Dwarf virus (genus Luteovirus) from wild oats (Avena fatua), which reduced the abundance of the spillover host species, Setaria, allowing the wild oat to maintain ecological dominance (Power and Mitchell, 2004). Although disease dynamics may appear similar, and feedback into dilution or amplification effects, apparent competition describes the effect of pathogen prevalence on hosts, whereas, dilution and amplification describe the effects of host diversity on pathogen prevalence. Apparent competition is typically only a property of DD systems, but may be possible in FD systems if the pathogen is not directly diluted by the less susceptible, spillover host(s).

Apparent mutualism, in contrast to apparent competition, can occur in FD systems when hosts have equal competence. In this case, the introduction of a novel host can reduce the disease prevalence in the original host community members by replacing contacts. There is no 'competition' as all hosts are equally affected by the pathogen (Holt and Bonsall, 2017), and all hosts benefit from the addition of additional hosts. Apparent mutualism differs from dilution, as dilution assumes difference in host competence, which apparent mutualism does not.

In summary, under assumptions of FD dynamics, increasing host diversity can result in apparent mutualism when hosts are equal in their competence, and dilution can occur when hosts have unequal competence. Under assumptions of DD dynamics, increasing diversity results in amplification, irrespective of differences in host competence, if each host adds to overall disease transmission. However, apparent competition is possible if hosts vary in competence and one host 'out-competes' the others through having a superior immune system (lower pathogen related mortality).

\subsection{Interspecific transmission}

Changing host community structure changes intraspecific and interspecific contact rates, and thus disease dynamics. The interspecific transmission rate determines the effect of species richness on the outbreak potential, $R_{0}$. Transmission competence determines a host's role as amplifier or diluter, as the persistence of a disease in multiple hosts is determined by the interspecific and intraspecific transmission dynamics (section 3.1.1). For example, jackals (Canis adustus) need to be frequently reinoculated by rabies virus from domestic dogs to support an infection in the population (Rhodes et al., 1998; Keesing et al., 2006) as their intraspecific transmission is too low to sustain endemic prevalence, and thus jackals more likely a diluter than an amplifier of rabies. There are many approximations for interspecific transmission, focusing on the ecology of hosts or the co-evolution of pathogens and hosts (for example by using matching-allele and gene-for-gene models, (Poullain and Nuismer, 2012). Here, we consider those that we think may be most promising for addressing questions on the disease-diversity relationship.

Interspecific transmission can be modeled in a WAIFW-matrix (Who Acquired Infection From Who), an $n$ by $n$ matrix (here called $W$ ) showing the transmission between species in an $n$-species system, where intraspecific transmission can be found on the diagonal, and interspecific transmission on the off-diagonals (Dobson and Foufopoulos, 2001; Diekmann et al., 2010):

$$
W=\left[\begin{array}{cccc}
\beta_{11} & \beta_{12} & \ldots & \beta_{1 n} \\
\beta_{21} & & & \\
\vdots & \ddots & & \vdots \\
\beta_{n 1} & & & \beta_{n n}
\end{array}\right]
$$

In this matrix, transmission can be defined as $\beta_{i, j}$, where $i$ is the receiving host and $j$ the donating host $(j$ infects $i$ ). This matrix can be used to calculate the community $R_{0}$, which determines the overall disease prevalence in the 
systems, and the contributions of each individual host (Dobson and Foufopoulos, 2001). One way to estimate the interspecific transmission rate is to take the average of the intraspecific transmission rates (Dobson, 2004):

$$
\beta_{i, j}=\alpha_{i, j} \frac{\beta_{i, i}+\beta_{j, j}}{2}
$$

where $\alpha_{i, j}$ is a scaling parameter to account for differences in transmission potential between species $i$ and $j$, and determines the magnitude of diluting and amplifying behaviour of each species in the system (Dobson, 2004). A similar approach can also be applied to contact rates, $\kappa$, as defined in $\beta=\kappa \cdot c$, if one wants to define $c$ separately for individual host species, rather than the community average (Anguelov et al., 2014). However, there can be asymmetrical transmission between hosts, for example Blancou and Aubert (1997) suggest that for a fox with rabies to infect another species, such as a dog or cat, requires a million times more virus particles than would be necessary to infect another fox (Ostfeld et al., 2008). Such asymmetries may be overlooked when using the average of the intraspecific transmission rates.

\subsection{Quantifying contacts}

Heterogeneity in contacts can drastically change the initial spread and final outbreak of a disease (Eames and Keeling, 2003). Craft (2015) argued that network models are required to accurately model wildlife populations. In wildlife diseases, for instance, identifying super-spreaders and their role in the network can be critical for intervention management. As example, in a simulation study of wild chimpanzees, Rushmore et al. (2014) show that vaccinating the most connected individuals reduces the vaccination threshold by $35 \%$ compared to random vaccination. New advances in biomonitoring methods can help in the construction of contact network models, capturing the complexity in interactions that many animals show, including territoriality, sociality, and individual variation in movements (Viana et al., 2014; White et al., 2017). These networks can also be useful if we wish to describe transmission between inter-connected populations. While we do not review this complexity here, we expand on some of these exciting methods in the supplementary material. However, obtaining the data to construct robust network models is research intensive, and simplifying assumptions of homogeneity are frequently adopted. As social networks homogenize over time (Cross et al., 2004), such simplifying assumptions might not be unrealistic. Nonetheless, new methods, such as use of camera-trapping of animals at aggregation sites, can be adopted to estimate intraspecific and interspecific contact rates (Barasona et al., 2017) and usefully inform SIR models. Ultimately, disease transmission is determined by contact rates, and thus defining these rates is critical for generating realistic projections of disease spread. 


\begin{abstract}
Box 4: Empirical approximations of intra-species transmission
Estimating $\beta$ directly: Because it is challenging to quantify transmission rates directly, often approximations are used. For instance, viral particles activate effector T-cells, and so data on infection load can be approximated by measuring T-cells from serological samples of animal hosts (Blaser et al., 2014; Almocera and Hernandez-Vargas, 2019). Individual infection status can be determined for viral pathogens by (q)PCR, and viral pathogen load can be used to derive transmission rates (Streicker et al., 2010; Blaser et al., 2014; Almocera and Hernandez-Vargas, 2019; McCallum et al., 2017). Similarly for macroparasites, egg (dispersal stage) counts can be used to infer transmission rates (McCallum et al., 2017). Genome sequencing is a promising technique for estimating transmission rates for bacterial pathogens, as was illustrated for Mycobacterium bovis, and can reveal spatial patterns of transmission (Biek et al., 2012). For viruses, we can adopt phylodynamic approaches to infer transmission rates from birth-death models (MacPherson et al., 2020). Basic host demography parameters, such as $\delta$ (host natural mortality) and $r$ (host birth rate) are easily available, and can be allometrically scaled with host body size, we can similarly scale $\beta$ (De Leo and Dobson, 1996). For vector transmitted diseases, surveys of vectors associated with hosts can give an approximation of the rate of spread of the disease, and may provide insights into interspecific transmission (Lu et al., 2010).
\end{abstract}

Estimating the probability of successful transmission, $c$ : Even when the contact structure of the population is known, for example through biological monitoring, it is still challenging to define the infectivity of a contact - the probability of successful transmission after contact, $c$ (Craft, 2015; White et al., 2017). One approach used in wildlife disease dynamics is to quantify the Secondary Attack Rate (SAR), which is the ratio of the number of exposed hosts that developed the disease to the number of exposed hosts that did not (Childs et al., 2007). To accurately calculate the SAR, a clear distinction must be made between primary and secondary cases. For sexually transmitted diseases, which follow FD dynamics, the probability of becoming infected after contact can be calculated using the binomial distribution (Childs et al., 2007). The maximum likelihood of this probability following a single contact is identical to the SAR. While estimating $c$ remains a challenge, compounded by the fact that most disease models summarize the process of transmission into a single parameter, it is important to capture accurately. These methods are described in further detail in the supplementary material.

\title{
4 Future challenges and conclusion
}

Defining the shape of the transmission function between species is challenging, and seemingly small differences can have dramatic effects on predictions from multi-species models (Dobson, 2004). Given current rate of biodiversity and habitat degradation worldwide, there is an urgent need for studies on the importance of how reduced diversity and environmental carrying capacity of wild species may influence the transmission rates of their pathogens, and how this might cascade to possible spillover events. Habitat fragmentation and a decrease in the carrying capacity of an ecosystem can both affect transmission dynamics (Childs et al., 2007; Lafferty and Holt, 2003), and at the interface between natural and converted landscape we can find increased interspecific transmission (Wolfe et al., 2005; Faust et al., 2018; Goldberg et al., 2008).

As most hosts and pathogens exist within multi-host systems, we need to better understand how transmission affects disease outbreaks in such systems. The next-generation matrix (Diekmann et al., 2010), described in section 3.2, which allows us to calculate the community $R_{0}$ and each host's relative contribution, is one promising framework for modelling the dynamics of multi-host pathogens and disease maintenance in the reservoir. However, the complexity in quantifying transmission, with asymmetries in interspecific rates and among potential hosts following spillover events (Wolfe et al., 2007; Auld et al., 2017), can make the application of such models fraught. Additional challenges include accounting for spatial heterogeneity and contact structure, although over longer timescales transmission dynamics may appear more homogeneous (Cross et al., 2004). However, as pathogen sharing is affected by the overlapping geographical ranges of hosts (Davies and Pedersen, 2008), investigating the effect of local versus global dynamics remains important. 
It is also becoming increasingly clear that the evolutionary relationship between species plays a role in disease transmission, with strong evidence of phylogenetic signal in the likelihood of pathogen sharing among hosts (Davies and Pedersen, 2008; Farrell et al., 2019; Streicker et al., 2019; Olival et al., 2017). It is likely, for example, that similarity in the immune defenses of closely related species due to the evolutionary conservation of the cellular, immunological, or metabolic traits, favours virus exchange between them (Kuiken et al., 2006; Streicker et al., 2010). Similar phylogenetic signature in pest and pathogen sharing is observed in plants (Gilbert and Webb, 2007; Gilbert et al., 2012; Parker et al., 2015; Ssebuliba and Davies, 2021), and phylogeny is also suggested to be a strong predictor of pathogen impact, with declining severity of the effect of the disease with increasing evolutionary distance between hosts (Gilbert et al., 2015; Gougherty and Davies, 2021). It would be relatively straightforward to include this information in our models. For instance, following Parker et al. (2015), we can define the probability of successful transmission, $c$, to be dependent on the evolutionary relationship between hosts. Assuming that intraspecies probability of successful transmission is $c_{i}=\lambda_{i}$, we can then simply define the probability of successful transmission of a pathogen from the recipient species, $i$, to the donor species $j, c_{i j}$, as:

$$
c_{i j}=\lambda_{j} \cdot \frac{1}{1+\psi P P d_{i j}}
$$

where $\psi$ is a scaling constant and $P P d$ the Pairwise Phylogenetic Distance between the two hosts (in Million Years), such that at low PPd (closely related species), $c \approx 1$. While the phylogenetic diversity (PD) of hosts - the sum of the evolutionary branch lengths connecting species - (Faith, 1992) can provide a useful predictor of disease prevalence (Rolland et al., 2012; Huang et al., 2013, 2014), the phylogenetic distance separating species may be a more useful metric for scaling the probability of interspecific transmission, and in predicting novel host shifts (Poullain and Nuismer, 2012).

Spillover events are the driver of zoonotic epidemics, including that which precipitated the COVID-19 pandemic, and pathogen transmission rate is our best predictors of future host shifts and disease emergence (Poullain and Nuismer, 2012). In this paper we decomposed transmission into its separate parameters, and show how these are involved in the outbreak process. We defined a general contact rate that can encompass almost all existing variations of the transmission functions, and describe how contact rate underlies each. 


\section{References}

Almocera, A. E. S. and Hernandez-Vargas, E. A. (2019). Coupling multiscale within-host dynamics and between-host transmission with recovery (SIR) dynamics. Mathematical Biosciences, 309(January):34-41.

Anderson, R. M. and May, R. M. (1982). Coevolution of Hosts and Parasites. Parasitology, 85(2):411-426.

Anderson, R. M., May, R. M., and Ng, T. W. (1992). A ge-dependent choice of sexual partners and the transm ission dynam ics of H IV in S ub -S ah aran Africa. pages 135-155.

Anguelov, R., Garba, S. M., and Usaini, S. (2014). Backward bifurcation analysis of epidemiological model with partial immunity. Computers and Mathematics with Applications, 68(9):931-940.

Antonovics, J. (2017). Transmission dynamics: Critical questions and challenges. Philosophical Transactions of the Royal Society B: Biological Sciences, 372(1719).

Auld, S. K., Searle, C. L., and Duffy, M. A. (2017). Parasite transmission in a natural multihost-multiparasite community. Philosophical Transactions of the Royal Society B: Biological Sciences, 372(1719):1-10.

Bar-On, Y. M., Phillips, R., and Milo, R. (2018). The biomass distribution on Earth. Proceedings of the National Academy of Sciences of the United States of America, 115(25):6506-6511.

Barasona, J. A., Vicente, J., Díez-Delgado, I., Aznar, J., Gortázar, C., and Torres, M. J. (2017). Environmental presence of mycobacterium tuberculosis complex in aggregation points at the wildlife/livestock interface. Transboundary and emerging diseases, 64(4):1148-1158.

Barlow, N. D. (1991). A spatially aggregated disease/host model for bovine tb in new zealand possum populations. Journal of applied ecology, pages 777-793.

Barlow, N. D. (2000). Non-linear transmission and simple models for bovine tuberculosis. Journal of Animal Ecology, $69(4): 703-713$.

Begon, M., Bennett, M., Bowers, R. G., French, N. P., Hazel, S. M., and Turner, J. (2002). A clarification of transmission terms in host-microparasite models: Numbers, densities and areas. Epidemiology and Infection, 129(1):147-153.

Biek, R., O’Hare, A., Wright, D., Mallon, T., McCormick, C., Orton, R. J., McDowell, S., Trewby, H., Skuce, R. A., and Kao, R. R. (2012). Whole Genome Sequencing Reveals Local Transmission Patterns of Mycobacterium bovis in Sympatric Cattle and Badger Populations. PLoS Pathogens, 8(11).

Bjørnstad, O. N., Finkenstädt, B. F., and Grenfell, B. T. (2002). Dynamics of measles epidemics: estimating scaling of transmission rates using a time series sir model. Ecological monographs, 72(2):169-184.

Blancou, J. and Aubert, M. (1997). Transmission of rabies virus: importance of the species barrier. Bulletin de L'academie Nationale de Medecine, 181(2):301-11.

Blaser, N., Wettstein, C., Estill, J., Vizcaya, L. S., Wandeler, G., Egger, M., and Keiser, O. (2014). Impact of viral load and the duration of primary infection on hiv transmission: systematic review and meta-analysis. $A I D S$ (London, England), 28(7):1021.

Childs, J. E., Mackenzie, J. S., and Richt, J. A. (2007). Wildlife and emerging zoonotic diseases: the biology, circumstances and consequences of cross-species transmission, volume 315. Springer Science \& Business Media.

Civitello, D. J., Allman, B. E., Morozumi, C., and Rohr, J. R. (2018). Assessing the direct and indirect effects of food provisioning and nutrient enrichment on wildlife infectious disease dynamics. Philosophical Transactions of the Royal Society B: Biological Sciences, 373(1745). 
Craft, M. E. (2015). Infectious disease transmission and contact networks in wildlife and livestock. Philosophical Transactions of the Royal Society B: Biological Sciences, 370(1669).

Cressler, C. E., McLeod, D. V., Rozins, C., Van Den Hoogen, J., and Day, T. (2016). The adaptive evolution of virulence: A review of theoretical predictions and empirical tests. Parasitology, 143(7):915-930.

Cross, P. C., Johnson, P. L., Lloyd-Smith, J. O., and Getz, W. M. (2007). Utility of R0 as a predictor of disease invasion in structured populations. Journal of the Royal Society Interface, 4(13):315-324.

Cross, P. C., Lloyd-Smith, J. O., Bowers, J. A., Hay, C. T., Hofmeyr, M., and Getz, W. M. (2004). Integrating association data and disease dynamics in a social ungulate: Bovine tuberculosis in African buffalo in the Kruger National Park. Annales Zoologici Fennici, 41(6):879-892.

Daszak, P., Cunningham, A. A., and Hyatt, A. D. (2000). Emerging infectious diseases of wildlife - Threats to biodiversity and human health. Science, 287(5452):443-449.

Davies, T. J. and Pedersen, A. B. (2008). Phylogeny and geography predict pathogen community similarity in wild primates and humans. Proceedings of the Royal Society B: Biological Sciences, 275(1643):1695-1701.

de Jong, M. C., Diekmann, O., and Heesterbeek, H. (1995). How does transmission of infection depend on population size. Epidemic models: their structure and relation to data, 5(2):84-94.

De Leo, G. A. and Dobson, A. P. (1996). Allometry and simple epidemic models for microparasites. Nature, $379(6567): 720-722$.

Dieckmann, U., Metz, J. A., and Sabelis, M. W. (2005). Adaptive dynamics of infectious diseases: in pursuit of virulence management. Number 2. Cambridge University Press.

Diekmann, O., Heesterbeek, J., and Roberts, M. G. (2010). The construction of next-generation matrices for compartmental epidemic models. Journal of the Royal Society Interface, 7(47):873-885.

Diekmann, O. and Kretzschmar, M. (1991). Patterns in the effects of infectious diseases on population growth. Journal of Mathematical Biology, 29(6):539-570.

Dobson, A. (2004). Population Dynamics of Pathogens with Multiple Host Species. 164(november).

Dobson, A. and Foufopoulos, J. (2001). Emerging infectious pathogens of wildlife. Philosophical Transactions of the Royal Society B: Biological Sciences, 356(1411):1001-1012.

Eames, K. T. and Keeling, M. J. (2003). Contact tracing and disease control. Proceedings of the Royal Society B: Biological Sciences, 270(1533):2565-2571.

Elguero, E., Délicat-Loembet, L. M., Rougeron, V., Arnathau, C., Roche, B., Becquart, P., Gonzalez, J.-P., Nkoghe, D., Sica, L., Leroy, E. M., et al. (2015). Malaria continues to select for sickle cell trait in central africa. Proceedings of the National Academy of Sciences, 112(22):7051-7054.

Faith, D. P. (1992). Conservation evaluation and phylogenetic diversity. Biological conservation, 61(1):1-10.

Farrell, M. J., Govender, D., Hajibabaei, M., Van Der Bank, M., and Davies, T. J. (2019). Bacterial diversity in the waterholes of the Kruger National Park: An eDNA metabarcoding approach. Genome, 62(3):229-242.

Faust, C. L., McCallum, H. I., Bloomfield, L. S., Gottdenker, N. L., Gillespie, T. R., Torney, C. J., Dobson, A. P., and Plowright, R. K. (2018). Pathogen spillover during land conversion. Ecology Letters, 21(4):471-483.

Fenton, A. and Pedersen, A. B. (2005). Community epidemiology framework for classifying disease threats. Emerging Infectious Diseases, 11(12):1815-1821. 
Fenton, A., Streicker, D. G., Petchey, O. L., and Pedersen, A. B. (2015). Are all hosts created equal? Partitioning host species contributions to parasite persistence in multihost communities. American Naturalist, 186(5):610-622.

Ferrari, M. J., Perkins, S. E., Pomeroy, L. W., and Bjrnstad, O. N. (2011). Pathogens, social networks, and the paradox of transmission scaling. Interdisciplinary Perspectives on Infectious Diseases, 2011.

Fine, A. E., Bolin, C. A., Gardiner, J. C., and Kaneene, J. B. (2011). A study of the persistence of mycobacterium bovis in the environment under natural weather conditions in Michigan, USA. Veterinary Medicine International, 2011.

Fromont, E., Pontier, D., and Langlais, M. (1998). Dynamics of a feline retrovirus (FeLV) in host populations with variable spatial structure. Proceedings of the Royal Society B: Biological Sciences, 265(1401):1097-1104.

Gandon, S. (2004). Evolution of multihost parasites. Evolution, 58(3):455-469.

Gilbert, G. S., Briggs, H. M., and Magarey, R. (2015). The impact of plant enemies shows a phylogenetic signal. PLoS ONE, 10(4):1-11.

Gilbert, G. S., Magarey, R., Suiter, K., and Webb, C. O. (2012). Evolutionary tools for phytosanitary risk analysis: Phylogenetic signal as a predictor of host range of plant pests and pathogens. Evolutionary Applications, 5(8):869878.

Gilbert, G. S. and Webb, C. O. (2007). Phylogenetic signal in plant pathogen-host range. Proceedings of the National Academy of Sciences, 104(12):4979-4983.

Goldberg, T. L., Gillespie, T. R., Rwego, I. B., Estoff, E. L., and Chapman, C. A. (2008). Forest fragmentation as cause of bacterial transmission among nonhuman primates, humans, and livestock, uganda. Emerging infectious diseases, 14(9):1375.

Gougherty, A. V. and Davies, T. J. (2021). Towards a phylogenetic ecology of plant pests and pathogens. Philosophical Transactions of the Royal Society B, 376(1837):20200359.

Graham, J. P., Leibler, J. H., Price, L. B., Otte, J. M., Pfeiffer, D. U., Tiensin, T., and Silbergeld, E. K. (2008). The animal-human interface and infectious disease in industrial food animal production: Rethinking biosecurity and biocontainment. Public Health Reports, 123(3):282-299.

Greer, A. L., Briggs, C. J., and Collins, J. P. (2008). Testing a key assumption of host-pathogen theory: Density and disease transmission. Oikos, 117(11):1667-1673.

Gubbins, S., Gilligan, C. A., and Kleczkowski, A. (2000). Population dynamics of plant-parasite interactions: Thresholds for invasion. Theoretical Population Biology, 57(3):219-233.

Halliday, F. W. and Rohr, J. R. (2019). Measuring the shape of the biodiversity-disease relationship across systems reveals new findings and key gaps. Nature Communications, 10(1):1-10.

Han, B. A., O’Regan, S. M., Paul Schmidt, J., and Drake, J. M. (2020). Integrating data mining and transmission theory in the ecology of infectious diseases. Ecology Letters, 23(8):1178-1188.

Han, B. A., Park, A. W., Jolles, A. E., and Altizer, S. (2015). Infectious disease transmission and behavioural allometry in wild mammals. Journal of Animal Ecology, 84(3):637-646.

Haydon, D. T., Cleaveland, S., Taylor, L. H., and Laurenson, M. K. (2002). Identifying reservoirs of infection: A conceptual and practical challenge. Emerging Infectious Diseases, 8(12):1468-1473.

Hoch, T., Fourichon, C., Viet, A. F., and Seegers, H. (2008). Influence of the transmission function on a simulated pathogen spread within a population. Epidemiology and Infection, 136(10):1374-1382. 
Hochberg, M. E. (1991). Non-linear transmission rates and the dynamics of infectious disease. Journal of theoretical biology, 153(3):301-321.

Holt, R. D. and Bonsall, M. B. (2017). Apparent Competition. Annual Review of Ecology, Evolution, and Systematics, 48:447-471.

Holt, R. D. and Pickering, J. (1985). Infectious Disease and Species Coexistence : A Model of Lotka-Volterra Form Author ( s ): Robert D . Holt and John Pickering Source : The American Naturalist, Vol . 126 , No . 2 ( Aug ., 1985 ), pp . 196-211 Published by : The University of Chicago Press. The American naturalist, 126(2):196-211.

Huang, Z. Y., de Boer, W. F., Van Langevelde, F., Xu, C., Ben Jebara, K., Berlingieri, F., and Prins, H. H. (2013). Dilution effect in bovine tuberculosis: Risk factors for regional disease occurrence in Africa. Proceedings of the Royal Society B: Biological Sciences, 280(1765):1-7.

Huang, Z. Y., Xu, C., Van Langevelde, F., Prins, H. H., Ben Jebara, K., and De Boer, W. F. (2014). Dilution effect and identity effect by wildlife in the persistence and recurrence of bovine tuberculosis. Parasitology, 141(7):981-987.

IPBES (2020). Workshop Report on Biodiversity and Pandemics of the Intergovernmental Platform on Biodiversity and Ecosystem Services. page 108.

Jones, K. E., Patel, N. G., Levy, M. A., Storeygard, A., Balk, D., Gittleman, J. L., and Daszak, P. (2008). Global trends in emerging infectious diseases. Nature, 451(7181):990-993.

Keeling, M. J. and Rohani, P. (2011). Modeling infectious diseases in humans and animals. Princeton university press.

Keesing, F., Belden, L. K., Daszak, P., Dobson, A., Harvell, C. D., Holt, R. D., Hudson, P., Jolles, A., Jones, K. E., Mitchell, C. E., Myers, S. S., Bogich, T., and Ostfeld, R. S. (2010). Impacts of biodiversity on the emergence and transmission of infectious diseases. Nature, 468(7324):647-652.

Keesing, F., Holt, R. D., and Ostfeld, R. S. (2006). Effects of species diversity on disease risk. Ecology Letters, $9(4): 485-498$.

Kermack, W. O. and McKendrick, A. G. (1927). A contribution to the mathematical theory of epidemics. Proceedings of the royal society of london. Series A, Containing papers of a mathematical and physical character, 115(772):700721.

Knell, R. J., Begon, M., and Thompson, D. J. (1996). Transmission dynamics of bacillus thuringiensis infecting plodia interpunctella: a test of the mass action assumption with an insect pathogen. Proceedings of the Royal Society of London. Series B: Biological Sciences, 263(1366):75-81.

Kuiken, T., Holmes, E. C., McCauley, J., Rimmelzwaan, G. F., Williams, C. S., and Grenfell, B. T. (2006). Host species barriers to influenza virus infections. Science, 312(5772):394-397.

Lafferty, K. D. and Holt, R. D. (2003). How should environmental stress affect the population dynamics of disease? Ecology Letters, 6(7):654-664.

Lloyd-Smith, J. O., Schreiber, S. J., Kopp, P. E., and Getz, W. M. (2005). Superspreading and the effect of individual variation on disease emergence. Nature, 438(7066):355-359.

Loreau, M. and Hector, A. (2001). Partitioning selection and complementarity in biodiversity experiments. Nature, $412(6842): 72-76$.

Lu, D. B., Wang, T. P., Rudge, J. W., Donnelly, C. A., Fang, G. R., and Webster, J. P. (2010). Contrasting reservoirs for Schistosoma japonicum between marshland and hilly regions in Anhui, China a two-year longitudinal parasitological survey. Parasitology, 137(1):99-110. 
MacPherson, A., Louca, S., McLaughlin, A., Joy, J. B., and Pennell, M. W. (2020). A general birth-death-sampling model for epidemiology and macroevolution. bioRxiv.

May, R. M. and Anderson, R. M. (1984). Spatial heterogeneity and the design of immunization programs. Mathematical Biosciences, 72(1):83-111.

McCallum, H. (2016). Models for managing wildlife disease. Parasitology, 143(7):805-820.

McCallum, H., Barlow, N., and Hone, J. (2001). How should pathogen transmission be modelled? Trends in Ecology and Evolution, 16(6):295-300.

McCallum, H., Fenton, A., Hudson, P. J., Lee, B., Levick, B., Norman, R., Perkins, S. E., Viney, M., Wilson, A. J., and Lello, J. (2017). Breaking beta: Deconstructing the parasite transmission function. Philosophical Transactions of the Royal Society B: Biological Sciences, 372(1719).

McKay, B., Ebell, M., Dale, A. P., Shen, Y., and Handel, A. (2020). Virulence-mediated infectiousness and activity trade-offs and their impact on transmission potential of influenza patients: Infectiousness and Activity Trade-Offs. Proceedings of the Royal Society B: Biological Sciences, 287(1927).

Miller, M. A., Kerr, T. J., de Waal, C. R., Goosen, W. J., Streicher, E. M., Hausler, G., Rossouw, L., Manamela, T., van Schalkwyk, L., Kleynhans, L., et al. (2021). Mycobacterium bovis infection in free-ranging african elephants. Emerging Infectious Diseases, 27(3):990.

Mollentze, N., Streicker, D. G., Murcia, P. R., Hampson, K., and Biek, R. (2020). Virulence mismatches in index hosts shape the outcomes of cross-species transmission. Proceedings of the National Academy of Sciences of the United States of America, 117(46):28859-28866.

Novozhilov, A. S. (2008). Heterogeneous Susceptibles-Infectives model: Mechanistic derivation of the power law transmission function. pages $1-14$.

Ogden, N. H. and Tsao, J. I. (2009). Biodiversity and Lyme disease: Dilution or amplification? Epidemics, 1(3):196206.

Olival, K. J., Hosseini, P. R., Zambrana-Torrelio, C., Ross, N., Bogich, T. L., and Daszak, P. (2017). Host and viral traits predict zoonotic spillover from mammals. Nature, 546(7660):646-650.

Ostfeld, R. S. and Keesing, F. (2000). Biodiversity series: the function of biodiversity in the ecology of vector-borne zoonotic diseases. Canadian Journal of Zoology, 78(12):2061-2078.

Ostfeld, R. S. and Keesing, F. (2012). Effects of host diversity on infectious disease. Annual Review of Ecology, Evolution, and Systematics, 43:157-182.

Ostfeld, R. S., Keesing, F., and Eviner, V. T. (2008). Infectious disease ecology: effects of ecosystems on disease and of disease on ecosystems. Princeton University Press.

Park, A. W., Farrell, M. J., Schmidt, J. P., Huang, S., Dallas, T. A., Pappalardo, P., Drake, J. M., Stephens, P. R., Poulin, R., Nunn, C. L., and Davies, T. J. (2018). Characterizing the phylogenetic specialism-generalism spectrum of mammal parasites. Proceedings of the Royal Society B: Biological Sciences, 285(1874).

Parker, I. M., Saunders, M., Bontrager, M., Weitz, A. P., Hendricks, R., Magarey, R., Suiter, K., and Gilbert, G. S. (2015). Phylogenetic structure and host abundance drive disease pressure in communities. Nature, 520(7548):542544 .

Pope, L. C., Butlin, R. K., Wilson, G. J., Woodroffe, R., Erven, K., Conyers, C. M., Franklin, T., Delahay, R. J., Cheeseman, C. L., and Burke, T. (2007). Genetic evidence that culling increases badger movement: implications for the spread of bovine tuberculosis. Molecular Ecology, 16(23):4919-4929. 
Poullain, V. and Nuismer, S. L. (2012). Infection genetics and the likelihood of host shifts in coevolving host-parasite interactions. American Naturalist, 180(5):618-628.

Power, A. G. and Mitchell, C. E. (2004). Pathogen spillover in disease epidemics. the american naturalist, 164(S5):S79-S89.

Rhodes, C. J., Atkinson, R. P. D., Anderson, R. M., and Macdonald, D. W. (1998). Rabies in Zimbabwe : reservoir dogs and the implications for disease control. (November 1996).

Roberts, M. G. and Heesterbeek, J. A. (2018). Quantifying the dilution effect for models in ecological epidemiology. Journal of the Royal Society Interface, 15(140).

Rohani, P., Green, C. J., Mantilla-Beniers, N. B., and Grenfell, B. T. (2003). Ecological interference between fatal diseases. Nature, 422(6934):885-888.

Rolland, J., Cadotte, M. W., Davies, J., Devictor, V., Lavergne, S., Mouquet, N., Pavoine, S., Rodrigues, A., Thuiller, W., Turcati, L., Winter, M., Zupan, L., Jabot, F., and Morlon, H. (2012). Using phylogenies in conservation: New perspectives. Biology Letters, 8(5):692-694.

Rudolf, V. H. and Antonovics, J. (2005). Species coexistence and pathogens with frequency-dependent transmission. American Naturalist, 166(1):112-118.

Rushmore, J., Caillaud, D., Hall, R. J., Stumpf, R. M., Meyers, L. A., and Altizer, S. (2014). Network-based vaccination improves prospects for disease control in wild chimpanzees. Journal of the Royal Society Interface, 11(97):20140349.

Sintayehu, D. W., Heitkönig, I. M., Prins, H. H., Tessema, Z. K., and De Boer, W. F. (2017). Effect of host diversity and species assemblage composition on bovine tuberculosis (bTB) risk in Ethiopian cattle. Parasitology, 144(6):783-792.

Smith, K. F., Goldberg, M., Rosenthal, S., Carlson, L., Chen, J., Chen, C., and Ramachandran, S. (2014). Global rise in human infectious disease outbreaks. Journal of the Royal Society Interface, 11(101):1-6.

Smith, M. J., Telfer, S., Kallio, E. R., Burthe, S., Cook, A. R., Lambin, X., and Begon, M. (2009). Host-pathogen time series data in wildlife support a transmission function between density and frequency dependence. Proceedings of the National Academy of Sciences of the United States of America, 106(19):7905-7909.

Ssebuliba, E. and Davies, T. J. (2021). Assessing the phylogenetic host breadth of millet pathogens and its implication for disease spillover. Ecological Solutions and Evidence, 2(1):1-11.

Streicker, D. G., Fallas González, S. L., Luconi, G., Barrientos, R. G., and Leon, B. (2019). Phylodynamics reveals extinction-recolonization dynamics underpin apparently endemic vampire bat rabies in Costa Rica. Proceedings of the Royal Society B: Biological Sciences, 286(1912).

Streicker, D. G., Fenton, A., and Pedersen, A. B. (2013). Differential sources of host species heterogeneity influence the transmission and control of multihost parasites. Ecology Letters, 16(8):975-984.

Streicker, D. G., Turmelle, A. S., Vonhof, M. J., Kuzmin, I. V., McCracken, G. F., and Rupprecht, C. E. (2010). Host phylogeny constrains cross-species emergence and establishment of rabies virus in bats. Science, 329(5992):676-679.

Tien, J. H. and Earn, D. J. (2010). Multiple transmission pathways and disease dynamics in a waterborne pathogen model. Bulletin of Mathematical Biology, 72(6):1506-1533.

Viana, M., Mancy, R., Biek, R., Cleaveland, S., Cross, P. C., Lloyd-Smith, J. O., and Haydon, D. T. (2014). Assembling evidence for identifying reservoirs of infection. Trends in Ecology and Evolution, 29(5):270-279. 
Vicente, J., Delahay, R. J., Walker, N. J., and Cheeseman, C. L. (2007). Social organization and movement influence the incidence of bovine tuberculosis in an undisturbed high-density badger Meles meles population. Journal of Animal Ecology, 76(2):348-360.

White, L. A., Forester, J. D., and Craft, M. E. (2017). Using contact networks to explore mechanisms of parasite transmission in wildlife. Biological Reviews, 92(1):389-409.

Wilber, M. Q., Langwig, K. E., Kilpatrick, A. M., McCallum, H. I., and Briggs, C. J. (2016). Integral Projection Models for host-parasite systems with an application to amphibian chytrid fungus. Methods in Ecology and Evolution, 7(10):1182-1194.

Wolfe, N. D., Daszak, P., Kilpatrick, A. M., and Burke, D. S. (2005). Bushmeat hunting, deforestation, and prediction of zoonotic disease. Emerging infectious diseases, 11(12):1822.

Wolfe, N. D., Dunavan, C. P., and Diamond, J. (2007). Origins of major human infectious diseases. Nature, $447(7142): 279-283$.

Woolhouse, M. E., Taylor, L. H., and Haydon, D. T. (2001). Population biology of multihost pathogens. Science, 292(5519):1109-1112.

\section{Tables}

\begin{tabular}{|c|c|c|c|}
\hline$F(S, I)$ & Description & units $\beta_{x}$ & Source \\
\hline & Homogeneous population & & \\
\hline$\frac{\beta_{F} S T}{N}$ & Simple frequency-dependence (FD) & $\mathrm{t}^{-1}$ & Anderson et al. (1992) \\
\hline$\frac{\beta_{A}^{N} S I}{A}$ & Simple density-dependence (DD) & ind ${ }^{-1} \mathrm{~m}^{2} \mathrm{t}^{-1}$ & Anderson and May (1982) \\
\hline$\beta_{D}^{A} S I$ & Simple DD with constant area & ind $^{-1} \mathrm{t}^{-1}$ & Anderson et al. (1992) \\
\hline$\beta_{q} S I N^{(q-1)}$ & Intermediate & ind $^{-q} \mathrm{t}^{-1}$ & Smith et al. (2009) \\
\hline$\frac{\beta_{F} S I}{K}$ & Carrying capacity dependence & $\mathrm{t}^{-1}$ & This manuscript \\
\hline$\beta_{F} \frac{\Lambda_{S I}}{N+X}$ & Asymptotic transmission & $\mathrm{t}^{-1}$ & Diekmann and Kretzschmar (1991) \\
\hline & Behavioural heterogeneity & & \\
\hline$\beta_{P L} S^{m} I^{n}$ & Power law & ind $^{1-m-n} \mathrm{t}^{-1}$ & Hochberg (1991); Novozhilov (2008) \\
\hline & Spatial heterogeneity & & \\
\hline$\beta_{D} I\left(N-\frac{I}{p}\right)$ & Refuge effect & ind $^{-1} t^{-1}$ & Barlow (1991) \\
\hline$\theta S \cdot \ln \left(1+\frac{\beta_{D} I}{\theta}\right)$ & Negative binomial & ind $^{-1} \mathrm{t}^{-1}$ & Barlow (2000); Greer et al. (2008) \\
\hline
\end{tabular}

Table 1: Different compositions of the variables $S$ and $I$ and the transmission rate, $\beta$, in the transmission function $(F(S, I))$ in traditional SIR models. Note that for each model $\beta$ assumes different values, depending on the units. See Table 2 for parameter definitions. 


\begin{tabular}{|c|c|c|}
\hline Parameter & Description & Unit \\
\hline$\beta_{x}$ & Transmission rate & See Table 1 \\
$\kappa$ & Contact rate (nr. individuals contacted per unit time) & $t^{-1}$ \\
$c$ & Probability of successful transmission & - \\
$\xi$ & Nr. of contacts & $m^{2}$ \\
$A$ & Area & - \\
$q$ & Contribution of 1 added individual for average $\kappa$ & ind $^{-1}$ \\
$X$ & Critical population size (half saturation time) & - \\
$m$ & Contribution of 1 S individual & - \\
$n$ & Contribution of 1 I individual & - \\
$p$ & Biotic/Abiotic factor & - \\
$\theta$ & Aggregation & ind $^{-1} \mathrm{t}^{-1}$ \\
\hline
\end{tabular}

Table 2: Parameters used in the transmission functions described in Section 2 and Table 1 and their corresponding units. The dimensions of parameter $\beta$ depend on the transmission function and are described in Table 1.

\begin{tabular}{|l|l|l|}
\hline Mechanism: & Dilution & Amplification \\
\hline FD & Direct: Encounter reduction & Indirect \\
\hline DD & Indirect & $\begin{array}{l}\text { Direct: Additive encounters } \\
\text { or selection effect }\end{array}$ \\
\hline
\end{tabular}

Table 3: Dilution and amplification occurring through various mechanisms. Varying host competence is assumed in all combinations, except in Amplification in DD pathogens (bottom right cell), where varying host competence is not required.

\section{Figures}




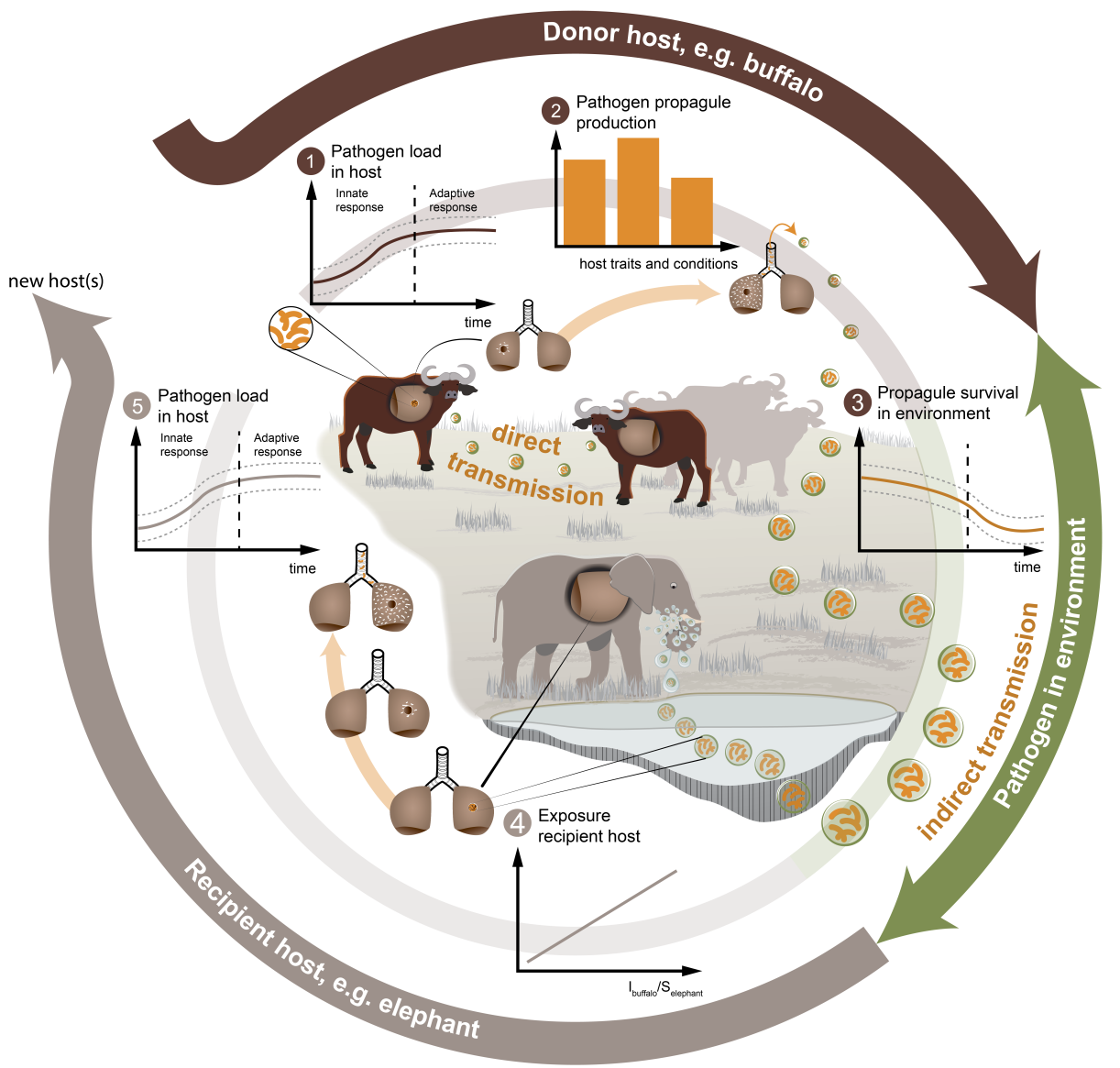

Figure 1: Spillover event of bovine Tuberculosis (bTB) from its reservoir, the African Buffalo, to a spillover host, the African Bush elephant. Graphs in figure show an approximation of the stages of transmission as described by McCallum et al. (2017). The figure includes both direct and indirect (environmental) transmission modes, typical for transmission of the bTB pathogen. In orange the progression of the mycobacterium is shown, in the lungs of both hosts, as well as their dispersal propagules. The figure gives an empirical example of the complexity of a pathogen's transmission cycle. Figure credit: Sylvia Herediaz, UBC Zoology. 


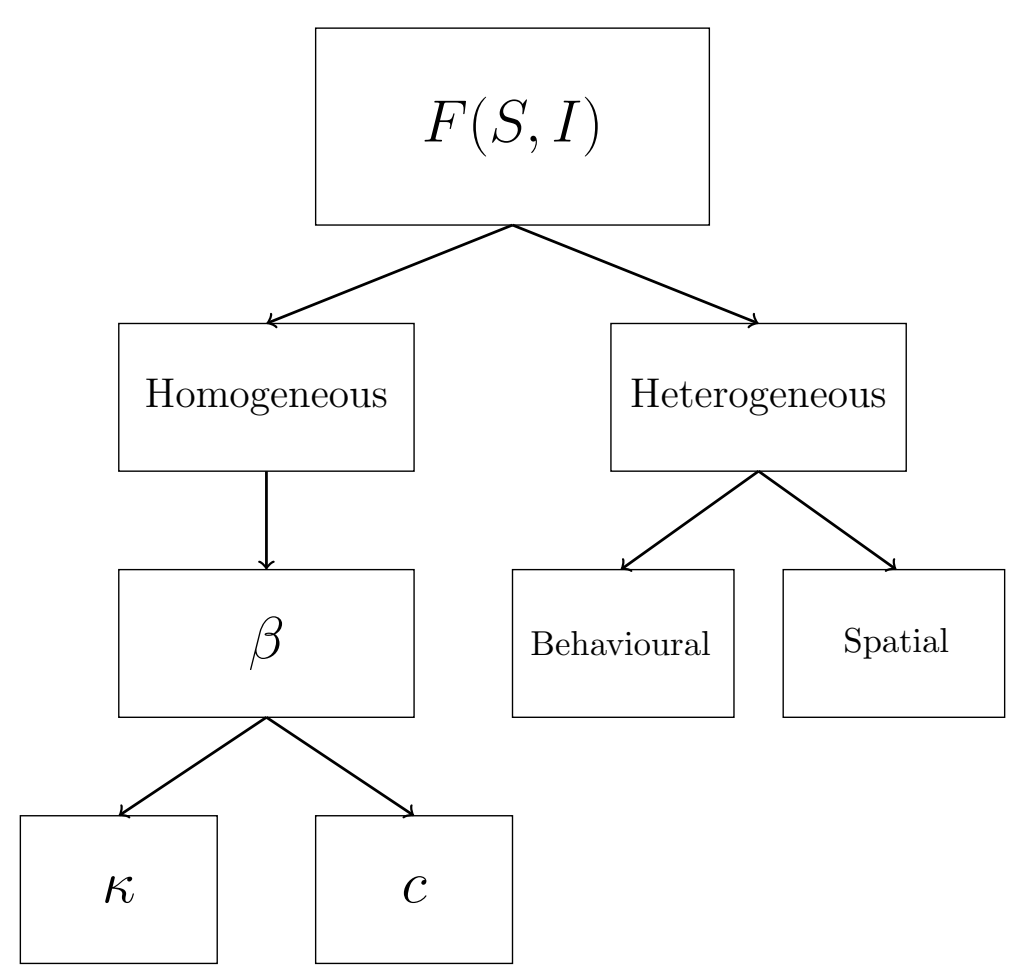

Figure 2: Graphical representation of the decomposition of the transmission function, $F(S, I)$, in traditional SIR compartmental disease models. $\beta$ is the transmission parameter, $\kappa$ the contact rate and $c$ the probability of successful transmission. On the left, we decompose the transmission parameter for homogeneously distributed host populations. On the right we show heterogeneously distributed host population, of which the dynamics are more complex and therefore the transmission function is not as simply decomposed. 


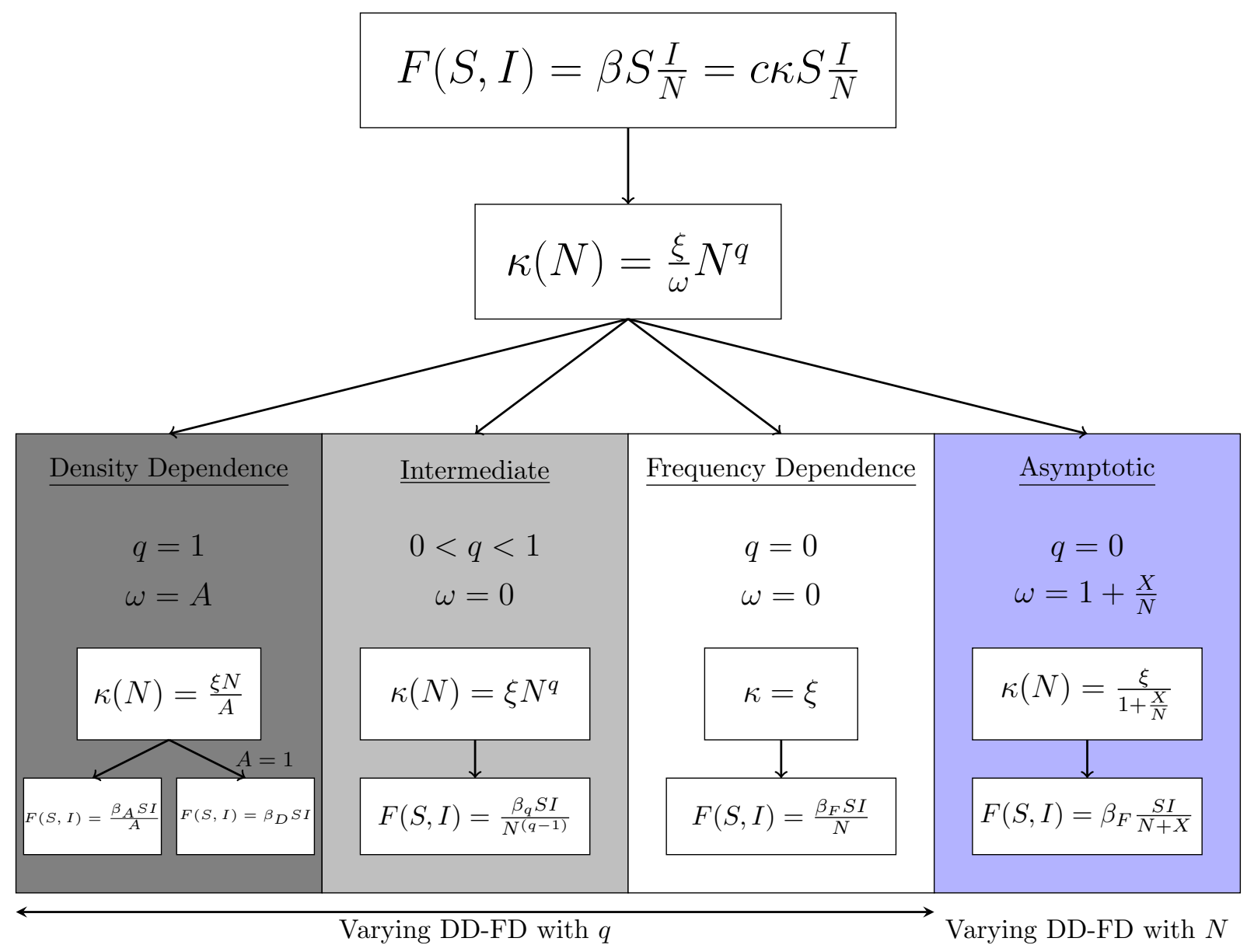

Figure 3: Flow chart showing how the contact rate function, $\kappa(N)$, determines the densityor frequency-dependent nature of the transmission function, $F(S, I)$. This in turn determines the dimensions of the transmission coefficient $\beta_{x}$. The first 3 panels show a spectrum from fully DD to FD, determined by parameter $q$. The right panel shows a transmission function that can vary from DD to FD with population size $N$. 


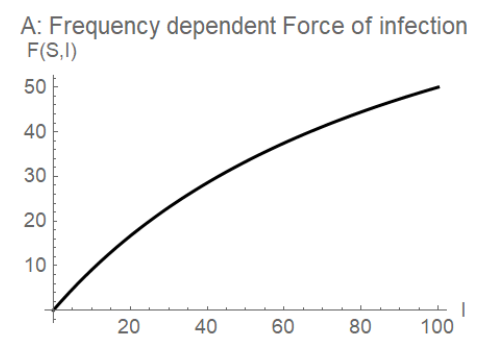

D: Carrying capacity dependent Force of infection

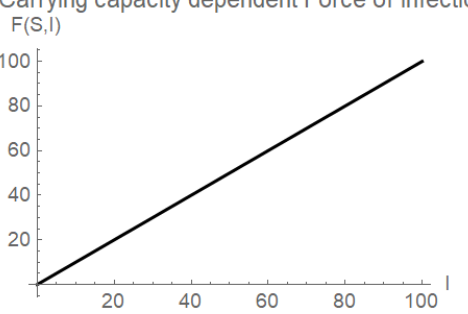

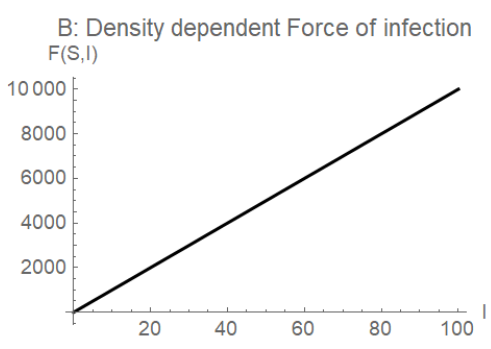

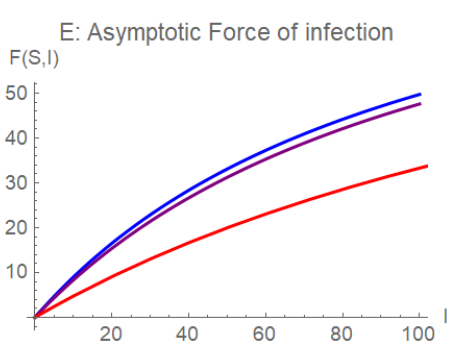

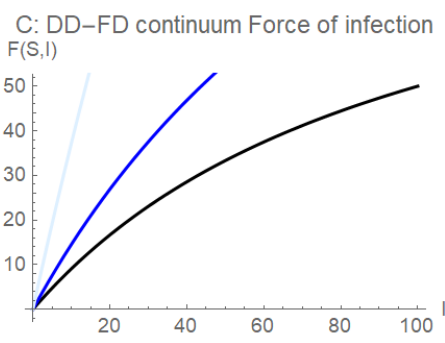

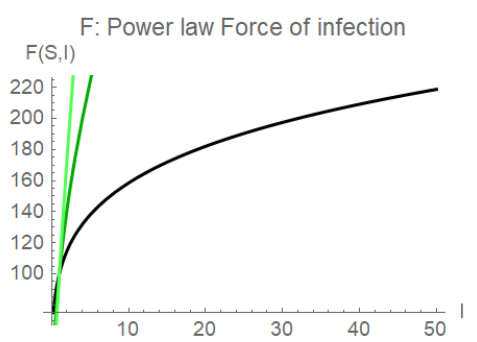

Figure 4: $\mathrm{F}(\mathrm{S}, \mathrm{I})$ in homogeneous populations with $S$ constant at 100 individuals. A: FD transmission. B: DD transmission C: Intermediate transmission. With light blue, $q=0.7$, in blue $q=0.9$ and black, $q=1$. D:Carrying capacity with $K=100$. E: Asymptotic transmission with in red $\omega_{0}=1$, in purple $\omega_{0}=10$ and in blue $\omega_{0}=100$. F: Power law transmission, black $n$ $=0.2$, dark green $n=0.5$, light green $n=0.8$.
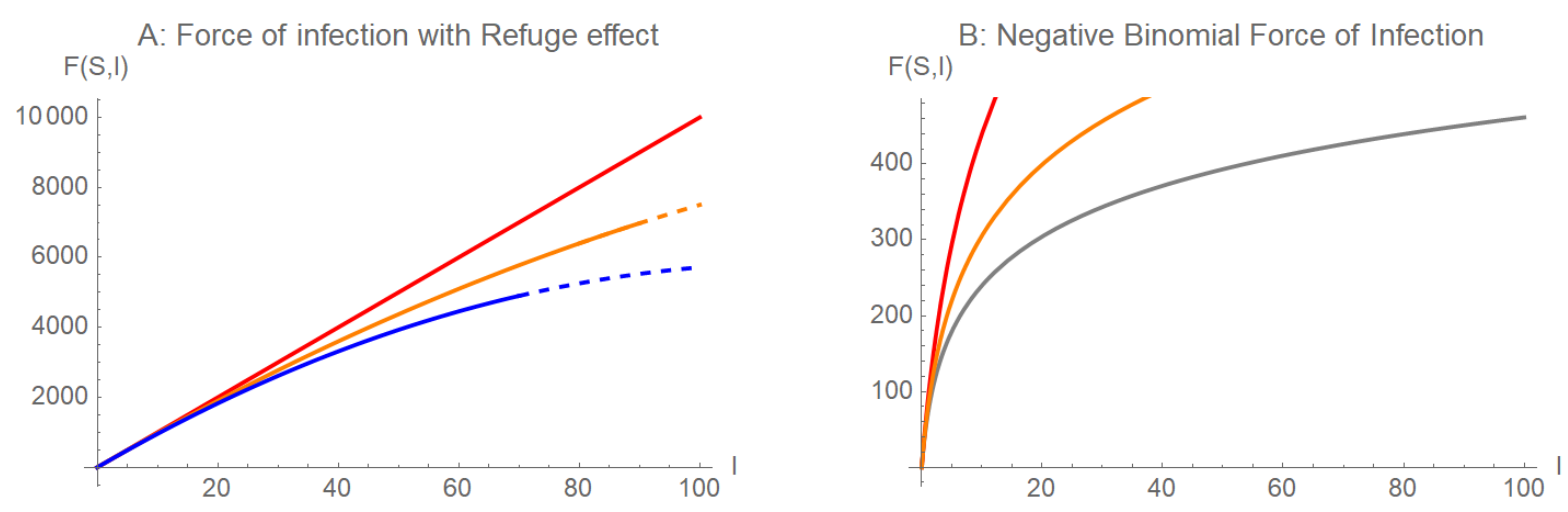

Figure 5: F(S,I) in spatially heterogeneous populations, $S$ set at 100. A: Refuge effect.Red, $q$ $=1$, orange $q=0.8$, blue $q=0.7$. Dashed line indicates the end of the biologically relevant transmission. B: Negative binomial. Gray, $k=1$, orange, $k=1.5$, red, $k=3$. 